\title{
Use of a Modified Ureteral Access Sheath (mUAS) in Semi-Rigid Ureteroscopy (URS) to Treat Large Upper Ureteral Stones is Associated with High Stone Free Rates
}

\author{
Jad Alsmadi ${ }^{1}$, Xiaohang $\mathrm{Li}^{2}$ and Guohua Zeng ${ }^{3 *}$ \\ ${ }^{1}$ Department of Urology, Minimally Invasive Surgery Center, The First Affiliated Hospital of Guangzhou Medical University, China \\ ${ }^{2}$ Guangzhou Institute of Urology, China \\ ${ }^{3}$ Guangdong Key Laboratory of Urology, China
}

*Corresponding author: Guohua Zeng, Guangdong Key Laboratory of Urology, \#1, kangda Road, Haizhu District, Guangzhou, Guangdong, China

Submission: 址 August 20, 2018; Published: 㭗 September 27, 2018

\begin{abstract}
Purpose: To examine differences in outcomes of semi-rigid-ureteroscopy (URS) with or without a modified-ureteral-access-sheath (mUAS) to treat large upper ureteral stones.

Methods: Patients with single, radio-opaque large upper ureteral stone $(\geq 10 \mathrm{~mm})$ treated using semi-rigid-URS between August 2013 and October 2016 were retrospectively evaluated. The stone-free (SF) status was determined from kidney-ureter-bladder-x-ray (KUB) films taken on postoperative day 1 and after 1 month.

Results: Of 103 patients meeting inclusion criteria, 43(41.75\%) and 60(58.25\%) were treated with semi-rigid-URS with and without mUAS, respectively. The immediate stone-free-rate (SFR) for the mUAS group was significantly higher than the non-mUAS group (40(93.0\%) vs. 46(76.7\%); $\mathrm{p}=0.033$ ). The SFR at 1 month was also high for patients treated using mUAS, but not statistically different from patients not treated with mUAS (41(95.3\%) mUAS vs. $51(85.0 \%)$ non-mUAS; $\mathrm{p}=0.115)$. Auxiliary procedure rates were significantly lower for mUAS patients compared to non-mUAS patients ( $4.65 \%$ vs. $23.34 \%$, respectively; $\mathrm{p}=0.01$ respectively). There were no significant differences in surgical duration and hospital stay, and the overall complication rates were statistically similar for both groups ( $2.3 \%$ vs. $5.0 \%$, for mUAS and non-mUAS, respectively; $\mathrm{p}=0.638$ ).
\end{abstract}

Conclusion: Application of mUAS to treat large upper ureteric stones was associated with higher immediate SFR and final SFR, and lower auxiliary procedure rates relative to patients treated without use of mUAS. Moreover, the use of mUAS did not lengthen operation duration or hospital stays.

Keywords: Ureteroscopy (URS); Semi-rigid; Large ureteral stones; Ureteral access sheath (UAS); Modified ureteral access sheath (mUAS)

Abbreviations: UAS/mUAS: Ureteral Access Sheath/Modified-UAS; URS: Ureteroscopy/Ureteroscope; F-URS: Flexible Ureteroscope; SFR: Stone Free Rate; KUB: Kidney-Ureter-Bladder X-Ray; NCCT: Non-Contrasted Computer Tomography Scan; US: Ultrasound; IVU: Intravenous Urography; RIRS: Retrograde Intrarenal Surgery; SWL: Shock Wave Lithotripsy; DJ: Double J Stent

\section{Introduction}

Semi-rigid ureteroscopy (URS) is the preferred technique for surgical management of ureteral stones. Semi-Rigid URS is a costeffective approach that offers low morbidity, short hospital stays, and a relatively low rate of additional procedures to achieve desired stone free rates (SFR). Upper ureteral stones, especially those that are large, have the lowest clearance rates as evidenced by rates of $77 \%, 85.4 \%$, and $80 \%$ observed in EAU guidelines [1], Kumar et al. [2], and Khairy Salem et al. [3], respectively. Meanwhile, a meta-analysis by Cui et al. [4] demonstrated higher initial SFR when comparing URS to Shock Wave Lithotripsy (SWL) to treat large upper ureteral stones. In order to achieve better treatment outcomes, several additions and modifications to procedures used to treat ureteral stones have been made. Anti-retropulsion devices increased ureteral stone clearance to between 98.5 and $100 \%$ in some series [5-7]. However, the routine use of anti-retropulsion devices remains an issue that is under debate [5-9]. The ureteral access sheath (UAS) positively impacted surgery times and stone management effectiveness while lowering intra-renal pressure [10$12]$, yet the routine use of this procedure is also a matter of debate [13]. In this study we examined whether applying suction to the UAS in semi-rigid URS is a valuable modification for treatment of large upper ureteral stones in terms of reduced SFR. 


\section{Materials and Methods}

\section{Patient enrollment}

After study approval by the institutional ethics committee, we retrospectively collected data for patients who underwent semirigid URS at our institution between August 2013 and October 2016. Patients having a single, radio-opaque upper ureteral stone $\geq 10 \mathrm{~mm}$ and who had not had a stent placed prior to the URS were enrolled. Children and patients having lower and middle ureteral stones, or radiolucent stones, as well as patients who had bilateral URS procedures to treat upper ureteral calculi, were excluded. Pneumatic Lithotripsy or laser lithotripsy were performed according to surgeon preference. A total of 103 patients treated with semi-rigid URS were included. Patient information, including age, sex, BMI, presence of preoperative UTI (on urine cultures), postoperative hemoglobin drop, stone size (largest diameter calculated on CT scan), stone sidedness, stone density in Hounsfield units, presence or absence of hydronephrosis, surgical time, use of a mUAS, lithotripsy mode (laser or Pneumatic Lithotripsy), the need for secondary procedures, presence of post-operative complications (graded according to the Clavien system) [14], and length of hospital stay (rounded to days from the operation day to discharge) were recorded. Patients with UTI were routinely treated for 3-5 days before the procedure. Stone Free status was evaluated at 24-48 hours (immediate SFR) by KUB and after 1 month (final SFR) using urinary tract non-contrasted-computer-Tomography (NCCT) or Intravenous-Urogram (IVU). Only 4 patients underwent IVU at the 1 month follow up; all were stone free on the initial KUB and were in the non-mUAS group. Patients were considered to be stone free when no stones or residual fragments $\leq 3 \mathrm{~mm}$ diameter were observed.

\section{mUAS design}

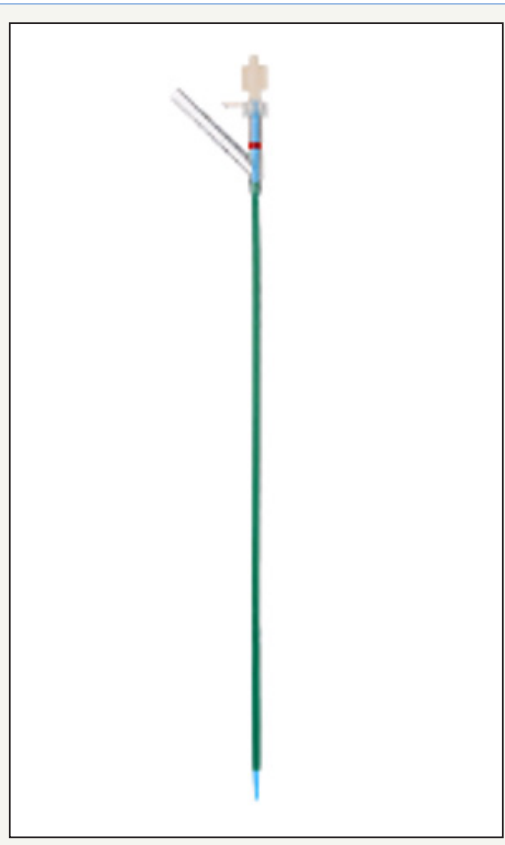

Figure 1
The mUAS was a standard UAS with an additional channel at the proximal end that allows the UAS to be connected to a suction machine. The mUAS is described in detail in our previous report [15]. The sheath consists of a straight distal segment and proximal bifurcated segments (Figure 1). The distal segment is a $35 \mathrm{~cm}$-long version for treatment of upper ureteral stones and can accommodate a $42-43 \mathrm{~cm}$ semi-rigid URS. The sheath is produced from the same material as that for a standard UAS and has a $12 \mathrm{Fr}$ and $14 \mathrm{Fr}$ inside and outside diameters, respectively. The bifurcated proximal segment is constructed of a straight and an oblique tube at a $45^{\circ}$ angle. Both the distal and proximal tubes have the same luminal diameter. A rubber seal with a center aperture is placed at the proximal end of the proximal straight tube. The longitudinal axis of the oblique branch includes a venting slit for regulating pressure (Figure 2). The proximal segment is $5.5 \mathrm{~cm}$ and constructed from clear plastic. The oblique sluice is connected to a negative pressure aspirator via transparent tubing. A red band is placed just proximal to the bifurcation for demarcation. An obturator that can be locked in place is used for sheath insertion.

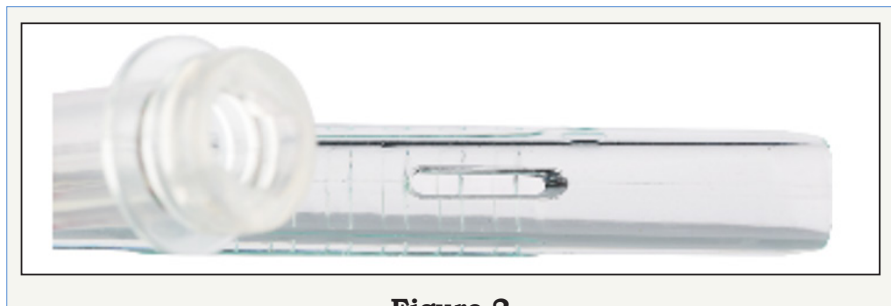

Figure 2

\section{Surgical procedure}

The mUAS was passed over a pre-inserted guidewire to a point just below the stone. The proper positioning of the sheath was verified with fluoroscopy prior to removal of the guidewire and the obturator. A rubber seal was placed and the oblique side branch was connected to a negative pressure aspirator running in continuous mode at $150-200 \mathrm{~mm} \mathrm{Hg}$. The aspirator was turned on to generate continuous pressurized irrigation before insertion of the ureteroscope. The semi-rigid URS was at least $3 \mathrm{Fr}$ smaller than the inner diameter of the mUAS and was passed through the rubber seal to maintain negative aspiration pressure. The scope was advanced just beyond the sheath. Pressurized irrigation was then connected to the scope and the flow was adjusted to $60-80 \mathrm{cc}$ per minute to create a continuous flow wherein irrigation emerging from the scope was immediately aspirated through the mUAS. Occasionally, the sheath had to be advanced several centimeters upward under direct vision, but typically the distal end of the sheath was placed within one centimeter of the stone. Lithotripsy was done using either holmium-YAG laser or a pneumatic lithotripsy probe. A double J (DJ) ureteral stent was routinely placed and was removed three to seven days after surgery. Patients were generally discharged on the first or second postoperative day after undergoing KUB. Intraoperative SF status was assessed both visually and fluoroscopically at the end of the procedure. The operative time was measured as the time from mUAS insertion to lithotripsy completion. Since we considered mUAS as an experimental device, active ureteral 
dilation was not performed for sheath insertion. If sheath insertion was unsuccessful after two attempts, this part of the procedure was abandoned and the patient was excluded from the study. For these patients, treatment of ureteral stones proceeded either without the sheath or with placement of a DJ stent. No additional devices or maneuvers, such as baskets or antiretropulsion devices, were used to increase the SFR in either treatment group.

\section{Results}

A total of 103 patients, 43 and 60 with and without mUAS, respectively, were enrolled. The mUAS group originally included 61 patients, but sheath insertion failed for 18(29.5\%); of those 12 were males; resulting in their exclusion, thus reducing the final enrollment to 43. Patient demographics (age, sex, BMI, DM, and hypertension) and stone sidedness were not significantly different between the two groups (Table 1). Stone sizes and densities were also similar between the mUAS and non-mUAS groups $112.66 \mathrm{~mm}$ vs. $14.94 \mathrm{~mm}$; 895.57 units vs. 776.47 units; $p=0.434$ vs. $p=0.275$; respectively). Preoperative UTI was more frequent in the mUAS group (11.6\% vs. $5 \%$ in the non-mUAS group) but this difference was not statistically significant $(\mathrm{p}=0.27)$. The presence and degree of hydronephrosis was evaluated using NCCT or IVU. Among the non-mUAS group, $86.67 \%$ showed a mild degree of hydronephrosis or none at all, whereas in the mUAS group the frequency of moderate to severe degree of hydronephrosis was significantly higher relative to the non-UAS group ( $45.5 \%$ vs. $13.34 \%$, respectively; $\mathrm{p}<0.001$ ). Operative and post-operative characteristics for both groups are described in (Table 2). Intraoperatively, the use of either pneumatic lithotripsy or laser was a matter of surgeon preference, and the rates of use were not significantly different between the two groups. The mean surgical duration was 3 min shorter for the mUAS group, but did not differ significantly from that for the non-mUAS group $(p=0.507)$. Post-operative complications assessed according to the
Clavien classification [14] were only minor, with Clavien's Grade 1 or 2, and were limited to postoperative fever and/or transient hematuria. All complications were managed by antipyretics and IV fluids, and some patients were given IV antibiotics. The complication rate of the mUAS group was lower than that of the non-mUAS group ( $2.3 \%$ vs. $5.0 \%)$, but this difference was not statistically significant $(\mathrm{p}=0.638)$. No intraoperative complications were reported and there were no instances of significant bleeding or need for blood transfusions in either group as assessed by postoperative hemoglobin drop measured by subtracting the postoperative value from the preoperative value $(p=0.236)$. SFRs were assessed by KUB at 24-48 hours as an immediate SFR for the procedures and at 1 month after the procedure using NCCT or IVU as the final SFR. Only 4 patients, all in the non-mUAS group, underwent IVU and all were stone free on the initial KUB done on the first post-operative day. The immediate SFR for the mUAS group was significantly higher than that for the non-mUAS group $(93.0 \%$ vs. 76.7\%; $p=0.033$ ). However, the final SFRs after 1 month for the mUAS and non-mUAS groups were not statistically different despite the high stone clearance rate seen for the mUAS group relative to the non-mUAS group ( $95.3 \%$ vs. $85.0 \%$, respectively; $\mathrm{p}=0.115$ ). Finally, decisions concerning the need for auxiliary procedures to achieve SF status were made either intraoperatively by visualizing stone retropulsion or by fluoroscopy, or postoperatively by KUB. Two patients in the mUAS group required retrograde intrarenal surgery (RIRS) as an adjunct procedure to remove large stone fragments that had migrated to intrarenal regions. In the non-mUAS group, 14 patients required extra management: 2 underwent RIRS and 12 underwent SWL during the same admission. The difference in auxiliary procedure rates was significantly lower for the mUAS group compared to the non-mUAS group (4.65\% vs. $23.34 \%$, respectively; $\mathrm{p}=0.01$ ).

Table 1: Patients and stone characteristics.

\begin{tabular}{|c|c|c|c|}
\hline & mUAS Group & Non-mUAS Group & p Value \\
\hline Number of patients & 43 & 60 & \\
\hline Mean age in years $( \pm S D)$ & $51.91 \pm 13.24$ & $48.2 \pm 11.04$ & 0.934 \\
\hline Sex ratio $(M / F)$ & $18 / 25$ & $24 / 36$ & 0.505 \\
\hline Mean BMI $(\mathrm{kg} / \mathrm{m} 2)( \pm \mathrm{SD})$ & $22.8 \pm 1.45$ & $23.95 \pm 1.67$ & 0.855 \\
\hline Having DM (number (\%)) & $1(2 \%)$ & $8(13 \%)$ & 0.076 \\
\hline Having hypertension (number (\%)) & $9(21 \%)$ & $12(20 \%)$ & 0.55 \\
\hline Laterality (Left/Right) & $28 / 15$ & $32 / 28$ & 0.311 \\
\hline Preoperative UTI (number (\%)) & $5(11.6 \%)$ & $3(5.0 \%)$ & 0.27 \\
\hline \multicolumn{4}{|c|}{ Hydronephrosis } \\
\hline No or mild, (number (\%)) & $23(53.5 \%)$ & $52(86.67 \%)$ & $<0.001$ \\
\hline Moderate-severe, (number (\%)) & $20(45.5 \%)$ & $8(13.34 \%)$ & $<0.001$ \\
\hline Stone Size (millimeter \pm SD) & $12.66 \pm 1.16$ & $14.94 \pm 1.82$ & 0.434 \\
\hline Stone Density (Hounsfield units) & $895.57 \pm 70.4$ & $776.47 \pm 120.09$ & 0.275 \\
\hline
\end{tabular}

mUAS: modified Ureteral Access Sheath, SD: Standard Deviation, M: male, F: Female, DM: Diabetes mellitus. 
Table 2: Operative and postoperative outcomes.

\begin{tabular}{|c|c|c|c|}
\hline & mUAS Group & $\begin{array}{c}\text { Non-mUAS } \\
\text { Group }\end{array}$ & p Value \\
\hline $\begin{array}{c}\text { Number of } \\
\text { patients }\end{array}$ & 43 & 60 & 0.033 \\
\hline $\begin{array}{c}\text { Number of } \\
\text { Stone Free } \\
\text { cases at 24-48 } \\
\text { hours (\%) }\end{array}$ & $40(93.0 \%)$ & $46(76.7 \%)$ & 0.115 \\
\hline $\begin{array}{c}\text { Number of } \\
\text { Stone Free cases } \\
\text { at 1 month (\%) }\end{array}$ & $41(95.3 \%)$ & $51(85.0 \%)$ & 0.69 \\
\hline $\begin{array}{c}\text { Surgery } \\
\text { mode Laser/ } \\
\text { Pneumatic } \\
\text { Lithotripsy }\end{array}$ & $23 / 17$ & $29 / 31$ & 0.105 \\
\hline $\begin{array}{c}\text { Hospital Stay } \\
\text { (days } \pm \text { SD) }\end{array}$ & $1.65 \pm 0.21$ & $1.95 \pm 0.29$ & 0.507 \\
\hline $\begin{array}{c}\text { Mean duration } \\
\text { of operation } \\
\text { (minutes } \pm \text { SD) }\end{array}$ & $38.19 \pm 9.23$ & $41.19 \pm 9.27$ & 0.638 \\
\hline $\begin{array}{c}\text { Auxiliary } \\
\text { Procedures } \\
\text { (number (\%)) }\end{array}$ & $2(4.65 \%)$ & $14(23.34 \%)$ & 0.01 \\
\hline $\begin{array}{c}\text { Postoperative } \\
\text { complications } \\
\text { (number (\%)) }\end{array}$ & $1(2.3 \%)$ & $3(5 \%)$ & $0.5 \pm 0.27$ \\
\hline $\begin{array}{c}\text { Mean } \\
\text { Hemoglobin } \\
\text { drop (g/l } \pm \text { SD) }\end{array}$ & $1.35 \pm 0.6$ & & 0.236 \\
\hline
\end{tabular}

mUAS: Modified Ureteral Access Sheath

\section{Discussion}

Urinary stone disease is becoming increasingly common and poses a significant health care burden for the working age population. Thus, the direct and indirect costs of kidney stones will likely continue to rise [16], which highlights the need for more prompt and cost-effective treatment methods. Semi-rigid URS to treat urinary stone disease remains as a readily available, durable, and low-cost treatment option, and provides high one-session SFR [17]. The overall success of URS for all ureteral stones is about $90 \%$ [18], whereas the success rate for semi-rigid URS to treat upper ureteral stones is only about 77\% [1]. As such, upper ureteral calculi, particularly large calculi, require special attention due to the relatively high rate of URS treatment failure, which is mainly due to stone retropulsion. The addition of flexible F-URS (RIRS) as an adjunct to semi-rigid URS achieved a total SFR of $90.5 \%$ for the management of upper ureteral stones [19], but this approach has a higher cost. On the other hand, the use of UAS facilitates multiple access to the upper urinary tract, decreases intrarenal pressure, improves surgeon vision by establishing a continuous outflow, and potentially reduces operating times [19]. Despite these benefits, and the potential for higher SFR, UAS has not been proven to yield a higher SFR [10-12]. Here this modification to the UAS (mUAS) allows for the addition of negative pressure or suction that further decreases intrarenal pressure and immediately suctions stone fragments to decrease the risk of retropulsion. Use of the mUAS resulted in higher SFR, without need for sophisticated equipment or additional costs. This study showed promising results for application of the mUAS, with an immediate and final SFR of $93.0 \%$ and $95.3 \%$, respectively, which is comparable to results obtained with antiretropulsion devices. Patients treated with the mUAS had a lower rate of auxiliary procedures. Most importantly, use of the mUAS does not require special training beyond that needed for use of standard UAS and has few additional costs. This study has several limitations. Patients for whom mUAS insertion failed were excluded, so the results reflect outcomes only for patients with successful mUAS insertion. Another limitation is the inconsistency of SFR assessment at 1 month. Most patients were evaluated by CT scan, but 4 patients in the non-mUAS arm underwent IVU. In addition to excluding radio-lucent stones because only KUB was done after 24-48 hours, we could not evaluate the immediate SFR in these patients. There were also deficiencies in some data in that stone density measurements (Hounsfield units) were not available for $49(48 \%)$ patients $(18(42 \%)$ in the mUAS group and $31(52 \%)$ in the non-mUAS group. Moreover, hemoglobin drop results were not available for some patients (25(24\%) overall, $9(21 \%)$ in the mUAS group and $16(27 \%)$ in the non-mUAS group. Stone composition was not available for $84(82 \%)$ patients and was not included in the final analysis. The degree of hydronephrosis was not evaluated using ultrasonic criteria, and instead was estimated using preoperative CT scan or IVU. Degree of hydronephrosis was divided into two categories (no-mild or moderate-severe) to decrease bias in the estimation. Finally, the patient sample was not randomized, although both patient and stone characteristics were balanced between the two groups. As a result, we conclude that applying suction to UAS, the mUAS, during relatively low cost semi-rigid URS procedures to treat large upper ureteral stones is effective and safe. Moreover, materials needed for mUAS are not sophisticated and thus provide an excellent working environment. The current data suggest that mUAS can be substituted for other SFR-enhancing measures. However, having flexible instruments as an adjunct to the procedure or for concomitant renal stones is still advisable. Further clinical trials using larger patient populations are needed to support these findings.

\section{Conclusion}

Applying suction to UAS, the mUAS, during relatively low cost semi-rigid URS procedures to treat large upper ureteral stones is effective and safe. Moreover, materials needed for mUAS are not sophisticated and thus provide an excellent working environment. The current data suggest that mUAS can be substituted for other SFR-enhancing measures. However, having flexible instruments as an adjunct to the procedure or for concomitant renal stones is still advisable. Further clinical trials using larger patient populations are needed to support these findings.

\section{Acknowledgement}

This work was financed by grants from the National Natural Science Foundation of China (No. 81370804 and No. 81670643), Guangzhou Science Technology and Innovation Commission (No. 201604020001, No. 201607010162 and No. 201704020193). 


\section{Conflict of Interest}

The authors declare that they have no conflict of interest.

\section{References}

1. Preminger GM, Tiselius HG, Assimos DG, Alken P, Buck C, et al. (2007) 2007 Guideline for the management of ureteral calculi. J Urol 178(6): 24182434 .

2. Kumar A, Nanda B, Kumar N, Kumar R, Vasudeva P, et al. (2015) A prospective randomized comparison between shockwave lithotripsy and semirigid ureteroscopy for upper ureteral stones $<2 \mathrm{~cm}$ : a single center experience. J Endourol 29(1): 47-51.

3. Khairy Salem H, El Ghoneimy M, El Atrebi M (2011) Semirigid ureteroscopy in management of large proximal ureteral calculi: is there still a role in developing countries? Urology 77(5): 1064-1068.

4. Cui X, Ji F, Yan H, Ou TW, Jia CS, et al. (2015) Comparison between extracorporeal shock wave lithotripsy and ureteroscopic lithotripsy for treating large proximal ureteral stones: A meta-analysis. Urology 85(4): 748-756.

5. Eisner BH, Dretler SP (2009) Use of the Stone Cone for prevention of calculus retropulsion during holmium:YAG laser lithotripsy: Case series and review of the literature. Urol Int 82(3): 356-360.

6. Gonen M, Cenker A, Istanbulluoglu O, Ozkardes H (2006) Efficacy of dretler stone cone in the treatment of ureteral stones with pneumatic lithotripsy. Urol Int 76(2): 159-162.

7. Cabrera FJ, Preminger GM, Lipkin ME (2014) Antiretropulsion devices. Curr Opin Urol 24(2): 173-178.

8. Kesler SS, Pierre SA, Brison DI, Preminger GM, Munver RJ (2008) Use of the escape nitinol stone retrieval basket facilitates fragmentation and extraction of ureteral and renal calculi: a pilot study. J Endourol 22: 1213-1218.

9. Yih Chyn P, Segaran S, Chew BH, Sriprasad S, Rane A (2016) Devices to help combat stone retropulsion during ureteroscopic lithotripsy in 2016. Journal of Clinical Urology 10(2): 87-92.
10. Kaplan AG, Lipkin ME, Scales CD, Preminger GM (2016) Use of ureteral access sheaths in ureteroscopy. Nat Rev Urol 13(3): 135-140.

11. L'esperance JO, Ekeruo WO, Scales CD, Marguet CG, Springhart WP, et al. (2005) Effect of ureteral access sheath on stone-free rates in patients undergoing ureteroscopic management of renal calculi. Urology 66(2): 252-255.

12. Kourambas J, Byrne RR, Preminger GM (2005) Does a ureteral access sheath facilitate ureteroscopy? J Urol 165(3): 789-793.

13. Berquet G, Prunel P, Verhoest G, Mathieu R, Bensalah K (2014) The use of a ureteral access sheath does not improve stone-free rate after ureteroscopy for upper urinary tract stones. World J Urol 32(1): 229232.

14. Dindo D, Demartines N, Clavien PA (2004) Classification of surgical complications: A new proposal with evaluation in a cohort of 6336 patients and results of a survey. Ann Surg 240(2): 205-213.

15. Zeng G, Wang D, Zhang T, Wan SP (2016) Modified access sheath for continuous flow ureteroscopic lithotripsy: A preliminary report of a novel concept and technique. J Endourol 30(9): 992-996.

16. Scales CD, Smith AC, Hanley JM, Saigal CS, Urologic Diseases in America Project (2012) Prevalence of kidney stones in the United States. European Urology 62(1): 160-165.

17. Türk C, Petř́k A, Sarica K, Seitz C, Skolarikos A, et al. (2016) EAU guidelines on interventional treatment for urolithiasis. Eur Urol 69(3): 475-482.

18. Assimos D, Krambeck A, Miller NL, Monga M, Murad MH, et al. (2016) Surgical management of stones: American urological association/ endourological society guideline, PART II. J Urol 196(4): 1153-1160.

19. Turkan S, Ekmekcioglu O, Irkilata L, Aydin M (2016) Is semirigid ureteroscopy sufficient in the treatment of proximal ureteral stones? When is combined therapy with flexible ureteroscopy needed? Springerplus 5: 30
Creative Commons Attribution 4.0

International License

For possible submissions Click Here

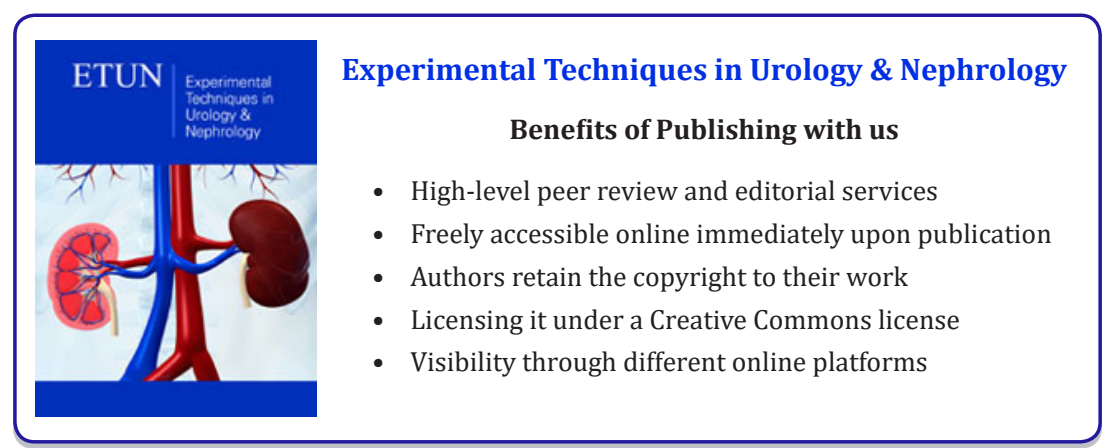

